\title{
Hypertexte, multimédia et inter-culture(s)
}

\section{Alain Cazade et Evelyn Perry}

\section{(2) OpenEdition}

\section{Journals}

Édition électronique

URL : http://journals.openedition.org/asp/4155

DOI : $10.4000 /$ asp. 4155

ISSN : 2108-6354

Éditeur

Groupe d'étude et de recherche en anglais de spécialité

\section{Édition imprimée}

Date de publication : 1 juillet 1994

Pagination : 107-124

ISSN : 1246-8185

\section{Référence électronique}

Alain Cazade et Evelyn Perry, « Hypertexte, multimédia et inter-culture(s) », ASp [En ligne], 4 | 1994, mis en ligne le 20 janvier 2014, consulté le 19 avril 2019. URL : http://journals.openedition.org/ asp/4155; DOI : 10.4000/asp.4155

Ce document a été généré automatiquement le 19 avril 2019

Tous droits réservés 


\title{
Hypertexte, multimédia et inter-culture (s)
}

\author{
Alain Cazade et Evelyn Perry
}

1 Cela fait de longues années que, au CERLACA-Dauphine comme partout ailleurs dans notre mouvance spécialisée assurément, nous nous posons la même question : comment faire passer chez nos étudiants une culture en langue anglaise, de nature générale et spécifique, d'une manière qui soit à la fois vivante, dynamique, attrayante, mais aussi riche et, de ce fait, quelque peu chargée de précision et de technicité dans tous les domaines? La réponse que nous avons trouvée est liée très intimement aux récents développements des technologies nouvelles tournant autour de l'informatique.

\section{Historique du projet}

2 Le CERLACA bénéficie d'un investissement de nombreuses années dans le domaine des langues et des technologies nouvelles. Cela nous a permis d'aboutir à diverses constatations.

Il existe différents types de logiciels mais certains, très simples, sont souvent, par rapport à d'autres, beaucoup plus compliqués, d'un rapport qualité/prix très intéressant - dans l'expression «rapport qualité/prix », entendons aussi : rapport investissement en temps d'apprentissage/efficacité pédagogique. Il existe différents types d'apprenants : doués ou moins doués, systématiques ou synthétiques, impatients de trouver ou chercheurs têtus, rapides ou appliqués... certains logiciels conviennent mieux que d'autres à tous les types apprenants! L'EAO semble plus efficace en travail de renforcement, de fixation de connaissances (passage d'une connaissance passive vers une connaissance active) qu'en découverte-intégration ou " acquisition $»^{1}$.

4 Le travail en langues aujourd'hui doit être multimédia. Le son est un élément indispensable, assez simple à manipuler et riche de possibilités à tous égards. Les images (fixes) sont très utiles, stimulantes, assez simples à gérer et à stocker. Les séquences vidéo offrent des contenus très riches; elles nécessitent un travail précis et long pour en faire 
digérer soit l'essentiel soit la totalité. Cela donne la tentation de créer ses propres séquences vidéo pour mieux cibler son champ d'action, avec les problèmes de temps, techniques et de budget que cela entraîne...

5 Nous ressentons de plus en plus le besoin d'adapter le contenu du travail proposé aux étudiants à l'enseignement pratiqué dans chaque filière, dans chaque UV, quelquefois dans différents groupes d'une même UV, d'année en année, etc.

6 Il est de plus en plus nécessaire de faire évoluer, rapidement et facilement, la nature du travail et du questionnement en fonction de sa réception par les apprenants: il est toujours très difficile de savoir d'emblée ce qu'il faut demander, comment il faut le demander, de repérer ce qui « marche » vraiment. Cela amène à des surprises quelquefois heureuses mais aussi malheureuses; il faut pouvoir supprimer ou modifier ce qui est malheureux et renforcer, développer ce qui est heureux.

Bref, le facteur temps est essentiel (ce qui sous-entend aussi le facteur simplicité) :

- le temps des apprenants : quand les faire travailler : en empiétant sur le cours, sur le temps de T.D. ou de T.P., en complément de cours : en libre-service, en tutorat semi-guidé... (avec quelle efficacité ?),

- le temps de l'enseignant: temps de réflexion préparatoire, temps de confection d'un exercice, temps de correction de l'exercice puis du ré-investissement dans le cours,

- le temps de latence entre le moment où un problème se fait jour en classe et celui où on devrait pouvoir lui donner une réponse dans un exercice, pour que le travail proposé reste en résonance avec la conscience, chez l'apprenant, de la difficulté rencontrée et de son contexte particulier.

8 Il faut avoir la possibilité de réduire ce temps à chaque étape, d'où la nécessité de pouvoir fabriquer un exercice rapidement en première instance, quitte à le faire évoluer par la suite. Aujourd'hui les outils donnant accès à la programmation « objet » et les avancées de la technologie multimédia nous donnent les moyens de répondre à nombre de ces exigences avec des moyens somme toute raisonnables, notamment l'environnement hypertexte.

9 C'est pourquoi Alain Cazade a décidé, sous le regard attentif et critique de Michèle Rivas², en bénéficiant de toutes les suggestions inspirées par le "native speaker's instinct» d'Evelyn Perry, avec l'expérience de nombreuses années de pratique de l'EAO à Dauphine et dans le secondaire ${ }^{3}$, dans la lancée d'un logiciel que nous utilisons depuis longtemps, présenté dans le cadre du GERAS il y a quelques années, Compulearn, de créer un nouvel outil. Il s'intitule : HELP YOURSELF et essaye de répondre à toutes les exigences exprimées plus haut.

10 HELP YOURSELF est écrit en langage hypertexte: LOGIIX (Infoaccess, anciennement $\mathrm{OWL}$ ). Il donne accès d'une part à toutes les richesses de l'environnement hypertexte : le non-séquentiel, la programmation « objet », la juxtaposition et l'échange de données de types les plus divers en simultané: textes, dictionnaires généraux ou/et lexiques spécialisés, textes de références, prises de notes, graphiques, sons (écoute et enregistrement), etc. Il offre d'autre part accès à des possibilités de questionnement et de traitement de réponses variées. 


\section{Choix pédagogiques}

11 Un certain nombre de choix pédagogiques ont nécessairement été faits. À ce stade, il faut insister sur le fait que, malgré les limites dues à la technique et aux aptitudes du concepteur à la maîtriser, la motivation pédagogique a toujours été l'élément initiateur et déterminant dans le développement de ce travail. Les choix en question sont les suivants.

1. Tout questionnement entraîne une production écrite, plus ou moins longue. Il ne suffit donc pas de cocher une case ici et là et donc de garder les yeux rivés sur l'écran, d'une manière servile ou/et hypnotisante. Le seul fait d'obliger le regard à quitter l'écran pour se concentrer sur le clavier permet de favoriser la mémorisation et la conceptualisation, tout en développant chez l'apprenant un espace transitaire de réflexion où le jugement personnel a davantage les moyens de jouer son rôle. On le voit, il n'est pas question ici de favoriser particulièrement le subliminal.

Le questionnement est à base de masquages-démasquages, une technique de questionnement très riche en possibilités de travail, inventée depuis longtemps mais qu'il fallait renouveler. On peut ainsi fabriquer des exercices de traduction, transformation de formes syntaxiques, concordance de temps, réordonnancement, classification ; toutefois, soulignons qu'on peut aussi, pour les amateurs irréductibles (et ils ont des raisons de l'être), fabriquer des QCMs simples, composés, à terme exclus, etc. Mais soulignons que si un QCM peut être très utile, il ne l'est que s'il est bien préparé, ce qui peut se révéler très onéreux en temps de préparation. Le rapport temps/efficacité est alors beaucoup moins avantageux qu'il n'y paraît de prime abord (voir le point 8 infra).

14 2. L'affichage du démasquage se fait à l'intérieur de n'importe quel texte, caractère par caractère, ceci afin de simuler la frappe du texte par l'apprenant (vitesse modulable). Tout d'abord l'affichage se fait en gras, pour attirer l'attention sur l'objet travaillé, puis l'affichage redevient normal : ceci afin de ne pas pervertir la lecture par la suite et donc pour protéger l'authenticité du texte qui reste affiché. Tout choix de fonte est possible, de 8 à... 50, ou plus encore si on veut, mais ce n'est guère raisonnable. Ceci nous donne la possibilité d'envisager même d'adapter un questionnement approprié à de très jeunes apprenants, en primaire éventuellement.

3. L'auteur du logiciel a voulu donner la possibilité de travailler sur des mots entiers, des expressions courtes ou longues, mais aussi sur des parties de mots ${ }^{4}$ : cela permet l'étude de préfixes, suffixes, racines communes, désinences, conjugaisons diverses, etc. mais aussi de travailler des modèles morpho-phoniques, morpho-sémantiques et des champs dérivationnels particuliers. On peut aussi tout simplement, de ce fait, suggérer une partie de la réponse pour cibler plus finement le niveau du travail ou tester des points plus pointus. Les possibilités d'un tel travail ne doivent être limitées en fait que par l'inventivité et les choix stratégiques de l'enseignant.

4. Trois différents types de remplacement du texte masqué sont accessibles:

a) --- de longueur fixe, permet de ne pas laisser deviner la longueur de l'expression recherchée,

b) $\ldots . . . ., \ldots . . .20 \% \ldots$..... ponctuation, espaces, chiffres et autres caractères symboliques (\% \$ $E=+\# *<>/:$ : ( ) §...) visibles,

c) _-_-_-_-_ tout est caché, pour des questions portant sur des données économiques, scientifiques, programme informatique, etc. 
17 5. Il a semblé indispensable de donner la possibilité de faire tout ce qui précède avec fichiers-« son », si on le désire, comme stimulus de départ, ce qui permet un travail de compréhension orale, de reconnaissance de formes syntaxiques, etc., avec en prime l'enregistrement de la voix de l'apprenant et la comparaison à un modèle donné. Cela permet un travail de prononciation. Pour développer davantage cet aspect, le concepteur a parallèlement prévu un module spécial simulant un environnement de laboratoire de langues, qui offre, en plus du laboratoire de langues classique, la stimulation de l'image et des possibilités de fixation de l'élément travaillé par l'adjonction de courtes séquences de questionnement écrit.

6. En cas de travail sur une partie de terme, l'apprenant doit frapper un mot complet, ceci pour favoriser la fixation d'éléments lexicaux réels et non celle de squelettes vides de sens. Néanmoins, nous avons tous constaté qu'en matière d'EAO, il fallait pousser l'apprenant à en faire toujours un peu plus, faute de quoi il se lasse et abandonne un peu trop vite. En l'incitant à poursuivre, on lui permettra peut-être de fournir au bout du compte une réponse qu'il n'aurait pas trouvée d'emblée. Pour faciliter ceci, un module de réponse de type « pendu » est constamment accessible qui permet de placer dans le corps d'une réponse attendue tous les petits éléments que l'on devine (ou que le fichier son a permis de reconnaître) $)^{5}$.

19 7. Il est possible, et la plupart du temps souhaitable, à partir d'un même texte de départ, de fabriquer plusieurs questionnements, portant sur des points de langue (fond ou/et forme), différents. Au fur et à mesure que le texte prend consistance dans la mémoire et la compréhension de l'apprenant, pour son contenu, on pourra développer plus finement le travail sur la matière de la langue. Le résultat de ce dernier travail, le plus délicat souvent, sera ainsi plus facilement ancré dans un contexte et l'on sait l'importance que ce point représente pour fixer la connaissance dans son intégrité.

8. Rapidité de fabrication pour les questionnements : pour répondre aux contraintes du facteur temps évoquées plus haut ; une question, sous sa forme élémentaire, se fabrique en deux « clicks » de souris à l'écran (une fois le texte de questionnement de départ déjà tapé depuis n'importe quel traitement de texte). On peut ensuite développer chaque point à volonté si on le désire.

9. Trois types de réponses sont pris en compte, entraînant un commentaire approprié si on a pris le temps de préparer celui-ci, sinon un commentaire par défaut est prévu.

a) Bonnes réponses (de qualité équivalente au texte initial) : l'apprenant entrant une de ces réponses prévues dans le questionnement verra celle-ci s'inscrire dans le texte, même si le texte original était différent. Ceci détermine le choix de telles réponses, choix délicat mais qui donne l'avantage de valoriser davantage la recherche de l'apprenant.

b) Réponses intéressantes mais de qualité inférieure : elles permettront au texte original de s'afficher mais auront également valorisé le travail de l'apprenant ; notation inférieure donc.

c) Mauvaises réponses (celles qu'il ne faut pas laisser passer !).

10. Log-book ${ }^{6}$ : Toutes les réponses de l'apprenant sont récupérées, avec chronodatage, quelles qu'elles soient, même incomplètes ou de type 'pendu' (avec l'état du masquerésultat dans ce cas). On peut faire de même pour les demandes de consultation de quelque document que ce soit. 
peut ainsi faire évoluer l'exercice en fonction des suggestions d'autres bonnes ou mauvaises réponses fournies par l'apprenant. On peut aussi, pour un travail de recherche, ou en vue de la mise au point d'un environnement de travail optimum, suivre l'évolution d'un apprenant, analyser des profils types de travail, d'apprenants, par rapport à un test psychologique fait au préalable, par exemple, analyser quel genre de caractère choisit tel genre de parcours : linéaire, circulaire, sédimentaire, complètement chaotique (mais que veut dire ce mot?).

Après avoir retracé l'historique et les grandes lignes de notre projet, nous voudrions maintenant esquisser le domaine interculturel autour duquel nous avons élaboré notre travail avant de présenter un certain nombre de nos choix pédagogiques.

En ce qui concerne l'aspect «culture» du projet, nous avons pris, comme champ de travail, la sous-culture noire américaine et, en particulier, l'image (ou plutôt les diverses images) de cette communauté projetée(s) dans la presse américaine. Si nous avons retenu le biais de la publicité, c'est en partie, bien sûr, parce que le langage de cette branche fait partie intégrante de l'enseignement de l'anglais de spécialité dispensé à Paris-Dauphine. Mais c'est aussi parce que la publicité, constituant en elle-même un vecteur transculturel, à visée socio-économique multiple, présente le reflet en condensé de toute la culture d'où elle émane. La publicité est également un domaine qui nous permet de travailler sur des documents iconographiques, traitant au même titre du texte et de l'image.

C'est en fonction de ces priorités que les différents écrans présentés dans notre projet d'Hypertexte ont été élaborés. Nous les passons rapidement en revue :

- Trois publicités (visuelles) tirées de la presse américaine, y compris des revues telles que Ebony ou Emerge, destinées principalement aux lecteurs noirs américains, mais achetées aussi par les blancs.

- Un texte court intitulé «Black Market » présentant l'évolution de l'image des noirs dans la publicité américaine, et faisant référence à l'importance croissante du pouvoir d'achat des consommateurs noirs aux États-Unis. Il bénéficie d'une aide lexicale en anglais et en français.

- Un texte de référence, tiré d'un numéro de Newsweek (août 1989), abordant les mêmes thèmes mais enrichi d'un nombre plus important d'exemples. Il bénéficie lui aussi d'une aide lexicale en anglais et en français.

- Un commentaire, accessible soit en anglais soit en français, sur les phénomènes culturels abordés dans le premier texte.

- Deux dictionnaires, mono et bilingue, couvrant les termes et les expressions les plus difficiles dans les trois textes.

- Un QCM (non noté) sur la prononciation et l'accent tonique des mots clés des trois textes. L'accès à l'élément son lui donne une bonne partie de son attrait et de son efficacité.

- Un exercice d'appui, de type 'simulation de laboratoire de langue', permet un travail encore plus poussé sur la prononciation, si on le désire, agrémenté de quelques exercices fabriqués avec HELP YOURSELF.

- Un autre test de type QCM (non noté) de compréhension, portant sur les trois textes cités auparavant.

- Un autre texte destiné à développer et analyser le thème des 'micromarchés' (appelé aujourd'hui : micro-marquétique), c'est-à-dire les créneaux ou segments du marché dont les paramètres sont définis en vue de cibler un public particulier. Ce texte est accompagné d'un exercice de masquage (noté, utilisant HELP YOURSELF) et d'un glossaire expliquant les notions clés. 
C'est avec ces outils de travail que l'apprenant abordera le carrefour culturel où se croisent la culture noire américaine, la publicité et la langue anglaise

\section{Travail du texte}

31 Voyons ensemble l'une des fenêtres que nous avons prévues pour le texte intitulé «Black Market $»^{7}$.

Fig. 1 Texte « Black Market»

\begin{tabular}{|c|c|c|c|c|c|}
\hline \multicolumn{6}{|c|}{ BLABK WMRKET } \\
\hline \multicolumn{6}{|c|}{$\begin{array}{l}\text { Introduction: Black Market } \\
\text { Gone are the days of including token blacks in McDonald's or Coke ads } \\
\text { just to placate the consciences of white middle-class Americans. Reaching } \\
\text { the black market is now a big business. Embarrassing "Jim Crow" } \\
\text { stereotypes, such as the fat old "Mammy" depicted on boxes of Aunt Jemima } \\
\text { pancake mix, have all but disappeared from advertising. } \\
\text { On today's billboards, TV commercials and product packaging, a new } \\
\text { range of trendier and, on the whole, more upmarket images of blacks are } \\
\text { projected. Marketers from both black- and white-owned advertising agencies } \\
\text { are combining elements of black music, language, and lifestyles to create ads } \\
\text { with a more "authentic" feel to them. This they do in the hope of capturing a } \\
\text { bigger chunk of the ever-increasing black market. } \\
\text { Ad executives claim that young whites and blacks alike identify with the } \\
\text { hip image conveyed by using black "iive talk" and well-known pop culture } \\
\text { personalities from the black community. }\end{array}$} \\
\hline Contents & d) & & & $\begin{array}{l}\text { Reforence Fext } \\
\text { Aunt Jemina? }\end{array}$ & \\
\hline & $\begin{array}{l}\text { nderstanding } \\
\text { he text - MCa }\end{array}$ & & & & \\
\hline
\end{tabular}

À partir de ce texte assez dense, l'apprenant découvre des notions de base sur la représentation des noirs dans la publicité américaine. En effet, pour l'étudiant d'un niveau moyen/moyen-fort, ce texte court est truffé de termes inconnus. Lorsque celui-ci bute contre un mot ou une expression difficile, il peut avoir recours à un minidictionnaire, préparé par nos soins. Si l'utilisateur clique sur la gauche du mot en question, il obtient une définition en anglais. S'il clique à droite, il en trouve une traduction française. Nous aurions pu proposer à l'apprenant une double fenêtre où il verrait les explications en français et en anglais simultanément. Si nous avons volontairement écarté cette possibilité, c'est pour favoriser la démarche de l'étudiant prêt à chercher un renseignement d'abord dans la langue cible. Cela vise aussi à renforcer une étape de mémorisation de la connaissance dans la langue cible, avant qu'elle ne se fixe dans la langue de référence, par une étape, aussi courte soit-elle, dans la mémoire de l'apprenant.

L'étudiant qui cliquera, par exemple, à gauche de l'expression: «Jim Crow stereotypes", trouvera l'explication suivante en anglais qui apparaitra dans un encadré :

Jim Crow stereotypes: unflattering stereotypes of blacks as shuffling, goodhumoured, bumbling idiots; also strongly associated with a type of racist joke belittling blacks.

Si toutefois, il reste toujours perplexe devant ces informations, l'étudiant pourra consulter la définition en français : 
Jim Crow stereotypes : nom donné de manière dépréciatrice et même raciste à un noir, stéréotype du noir au large sourire, bavard et simplet ; équivalent possible :

\section{Travail simulant un laboratoire de langue}

42 Si à tout moment l'étudiant a envie de faire un travail complémentaire sur l'accentuation, il pourra cliquer sur le bouton «Lab Work» en haut de l'écran pour se retrouver immédiatement dans la partie « laboratoire de langue » du logiciel.

Dans ce module, l'étudiant aura la possibilité d'écouter et répéter plusieurs phrases, toujours en relation avec le thème de la publicité. On peut écouter et repasser une même phrase un nombre illimité de fois.

Pour rendre le travail de laboratoire plus intéressant, nous avons également créé, avec HELP YOURSELF, un exercice de démasquage. L'utilisateur regarde la version écrite d'une phrase. Dans cette version il y a un ou plusieurs mot(s) caché(s). Après avoir écouté la 
phrase en question, l'apprenant peut enregistrer sa phrase et il pourra, bien sûr, la réécouter. Il pourra également repasser l'enregistrement du programme pour évaluer ses propres performances.

Lorsque tous les démasquages prévus sur toutes les phrases auront été effectués (de préférence, mais plus tôt s'il le désire), il pourra retravailler sur la prononciation de toutes les phrases en session de travail «automatisée », comme dans un laboratoire de langue traditionnel. Un autre travail en laboratoire pourrait isoler les éléments constitutifs d'une phrase en suivant une technique de type «back-building».

Ce travail de nature double, avec production écrite partielle et travail de compréhensionprononciation traditionnel, permet une séquence d'étude en mode libre-service, ou en tutorat semi-guidé, plus stimulante, pensons-nous, que ne le permettrait un matériel de laboratoire de langue habituel, du fait du travail de démasquage justement.

\section{Publicités trouvées dans la presse américaine}

Dans cette partie du logiciel, l'étudiant pourra analyser un document iconographique emprunté au monde de la publicité destinée aux noirs-américains. Parmi les publicités que nous avons retenues, on trouvera en annexe 1 un exemple de type pro bono publico qui vante les mérites de la lecture tout en encourageant la lutte contre l'illettrisme ${ }^{8}$. L'apprenant peut cliquer sur une partie de l'image qui l'intéresse pour faire venir un encadré fournissant des explications culturelles et/ou linguistiques.

Si l'utilisateur clique sur le titre « GET 'EM BOOKED NOW », il verra l'explication du jeu de mots «hooked/booked», une explication donnée d'abord en anglais, avec ensuite des traductions des mots clés en français.

"Get'em booked now": play on words: booked / hooked: Get these youngsters "booked" (hooked on books or interested in books, educated) before they get "hooked" on (addicted to) drugs. (booked=cultivé, scolarisé, formé par les livres..., hooked=prisonnier de... la drogue)

49 S'il clique sur le livre souriant en bas de page, il obtiendra une interprétation culturelle de cette image : les créateurs de cette page publicitaire ont en effet voulu mettre l'accent sur l'égalité entre citoyen blanc et citoyen noir en dessinant un livre moitié blanc, moitié noir. Par la même occasion, ils auront voulu créer l'impression, statistiquement fausse, que le problème d'illettrisme touche presque autant de blancs que de noirs.

Smiling book: half black, half white: suggesting the goals of racial equality and reinforcing the notion that illiteracy is as common amongst whites as it is amongst

blacks. Statistically, however, this is inaccurate.

Exemple d'utilisation conjointe de boutons son et de masquages, permise par «help yourself $»$.

51 L'encadré suivant («Niche marketing» 1) montre un écran type composé avec HELP YOURSELF, et intégré ici dans l'hypertexte «Black Market ». En cliquant sur le signe : >, (de couleur rouge), l'apprenant peut entendre le fichier son complet du texte enregistré au préalable; les signes > (toujours de couleur rouge) lui permettent d'entendre des éléments partiels de ce fichier. Ces boutons «son» ont l'avantage de donner plus d'authenticité et de vie à l'exercice, en permettant de travailler en compréhension et en reconnaissance audio tout en gardant le texte devant les yeux. Partant, ils facilitent d'autant le travail de démasquage fourni en parallèle; toutefois, si ce travail se révélait trop simple à l'expérience, il serait toujours possible d'accroître la difficulté en 
transformant les masquages. Ici, les masquages révèlent le nombre de caractères manquant; on pourrait tout aussi bien choisir le mode "masque de longueur fixe » :- , plus sibyllin, ou encore cacher davantage d'éléments. On pourrait fabriquer également un écran hypertexte avec uniquement les boutons "son » puis d'autres avec les masquages mais sans boutons «son » : le passage de ceci à cela, si court soit-il, pourrait favoriser la mémorisation du texte (contenu et forme).

\section{Fig.2 BLACK MARKET}

Niche marketing 1. F Niche marketing 2

(Complete sound file:> - Ctrl Pause to stop)

In the 1950s and 1960s - and well into the 1970s - American advertising strategy was aimed at a mass market. Mr and Ms Typical Consumer were ......... *.. white, AngloSaxon and Protestant (WASP), to have a ...... income, and to live in the ....... with their two lovely children.

In the 1980s, marketing specialists rightly perceived that billions of potential American consumers could not be lumped together in a ................ The massmarket concept no longer corresponded to the reality of the $\ldots . . . . . . . .$.

Sizeable ethnic minorities - such as blacks and hispanics - as well as women and adolescents all constituted discreet niches or micro-markets, each with significant purchasing power. Each of these market segments could be .................. according to a set of identifiable consumer ...................... Each potential micro-market would have to be courted in different ways. Hence the gradual development of ................. in the mid- and late-1980s with advertising campaigns tailor-made for narrowly defined niche audiences.

Il est impossible de reproduire de manière convaincante de tels écrans, la gestion des couleurs, des menus "en ligne », le changement d'apparence du pointeur de la souris lorsqu'il passe, par exemple, sur un bouton «question » (il prend alors la forme d'un point d'interrogation encadré) ou sur un bouton "son" (il se transforme alors en «bulle» de bande dessinée), les messages sonores en commentaire des réponses, etc., tout cela contribue à l'équilibre ergonomique de l'environnement de travail et à sa qualité.

Dans l'écran suivant, on peut noter différents types de masquages incomplets, ce qui permet d'orienter en partie, autant qu'on le désire en fait, le travail de l'apprenant. Pour qu'il travaille sur le mot "billions ", inutile qu'il cherche à entrer "thousands " ou « hundreds »; il ne dispose plus ici, à dessein, de l'aide d'un bouton « son ». Aussi bien a-t-on laissé apparaître la première lettre du mot recherché ? La même stratégie a été retenue pour les autres masquages portant sur «WASP», "lumped together », " micro-marketing », « ethnic », " hispanics ", « niches », " purchasing ", « tastes » et « audiences ».

\section{Fig. 3 BLACK MARKET}

E Niche marketing 1. Niche marketing 2.

Starting in the 1950s - and going well into the 1970s - American advertising strategy was aimed at a mass market. $\mathrm{Mr}$ and Ms Typical Consumer were presumed to be

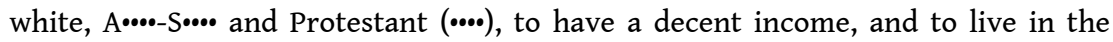
suburbs with their two lovely children.

In the 1980s, marketing specialists rightly perceived that b--- of potential American consumers could not be l...ed to...er in a single mass market. The --- market concept no longer corresponded to the reality of the market place.

Sizeable ....ic minorities - such as blacks and h.....ics - as well as women and adolescents constituted discreet ---s or micro-markets, each with significant --ing power. Each of these market segments could be defined and exploited according 
to a set of identifiable consumer ---es and preferences. Each potential m.... market would have to be courted in different ways. Hence the gradual development of micro-marketing in the mid- and late-1980s with advertising campaigns tailormade for narrowly defined niche au.......s. réponses sur le mot "purchasing", effectuées en mode "pendu ${ }^{9} .{ }^{10}$ Une tentative semblable a été faite sur le mot « niches».

\section{Fig. 4 BLACK MARKET}

E Niche marketing 1. Niche marketing 2.

Starting in the 1950s - and going well into the 1970s - American advertising strategy was aimed at a mass market. $\mathrm{Mr}$ and Ms Typical Consumer were presumed to be white, A....-S... and Protestant (....), to have a decent income, and to live in the suburbs with their two lovely children.

In the 1980s, marketing specialists rightly perceived that b--- of potential American consumers could not be l...ed t.....er in a single mass market. The --- market concept no longer corresponded to the reality of the market place.

Sizeable ....ic minorities - such as blacks and h....ics - as well as women and adolescents constituted discreet ni---s or micro-markets, each with significant puch--ing power. Each of these market segments could be defined and exploited according to a set of identifiable consumer ----es and preferences. Each potential m... - market would have to be courted in different ways. Hence the gradual development of micro-marketing in the mid- and late-1980s with advertising campaigns tailor-made for narrowly defined niche au.......s.

\section{« Log-book » ou « fichier-trace »}

L'extrait de tableau qui suit est emprunté au «log-book» - ou «fichier-trace » - qui est automatiquement alimenté par le logiciel à chaque fois que l'apprenant répond à une question ou ouvre un document particulier. On peut ainsi suivre l'évolution du travail de tout apprenant, récupérer sa notation éventuellement, corriger les questionnements en fonction des difficultés rencontrées... Du point de vue de la recherche, ces traces permettent également d'étudier le comportement cognitif des différents apprenants, si l'on analyse la progression des tâtonnements, les requêtes d'informations supplémentaires ici et là, les avancées et retours de question en question, de document en document On notera en ligne 2 la réponse incorrecte pour cause de majuscule oubliée. Elle aura pourtant été reconnue (signe \#, alors que + indique plus bas une bonne réponse), mais accompagnée d'un commentaire recommandant de commencer une phrase par une majuscule. Pour un nom géographique, l'absence de majuscule aurait été considérée comme erronée. Les signes nn indiquent des réponses en mode "pendu ", l'image du masque résultat est visible dans la colonne immédiatement à droite. En ligne 4, on remarquera aussi l'hésitation sur l'orthographe de "preferences » (un ou deux $r$ ?) : seul ce qui était sûr a été entré ; le mot " tastes " aura lui aussi posé quelque problème. On notera encore, en ligne 7, que l'apprenant aura cru reconnaitre le mot "niches " (entendu avec les boutons son de l'écran précédent), alors que « tastes » était attendu. À côté du masque résultat apparaissent, entre parenthèses, les caractères essayés par l'apprenant mais non reconnus dans le masque (signe : n-, et masque résultat:----es (ni)). Le même essai sera fructueux en ligne 8 , une minute plus tard. 
Fig. 5 Fichier trace

\begin{tabular}{|c|c|c|c|c|c|c|c|c|}
\hline 1 & Name & File & Date/time & Question item & sign & $\begin{array}{l}\text { learner's } \\
\text { answers }\end{array}$ & tries & pts \\
\hline 2 & $\begin{array}{l}\text { Nathalie } \\
\text { B. }\end{array}$ & EXEMPLES & $\begin{array}{l}\text { Mar } 2610: 29 \\
1994\end{array}$ & Developing & $\#$ & developing & $2 / 7$ & $8 / 10$ \\
\hline 3 & {$[\ldots]$} & {$[\ldots]$} & {$[\ldots]$} & {$[\ldots]$} & {$[\ldots]$} & {$[\ldots]$} & {$[\ldots]$} & {$[\ldots]$} \\
\hline 4 & $\begin{array}{l}\text { Nathalie } \\
\text { B. }\end{array}$ & BM & $\begin{array}{l}\text { Apr } 0918: 32 \\
1994\end{array}$ & $\begin{array}{ll}\text { tastes } & \text { and } \\
\text { preferences } & \end{array}$ & nn & $\begin{array}{ll}\cdots \cdots \cdots & \cdots \\
\text { prefer..... } & \end{array}$ & $1 / 13$ & $20 / 35$ \\
\hline 5 & $\begin{array}{l}\text { Nathalie } \\
\text { B. }\end{array}$ & BM & $\begin{array}{l}\text { Apr } 15 \text { 12:17 } \\
1994\end{array}$ & purchasing & nn & pu------ing & $1 / 5$ & $20 / 35$ \\
\hline 6 & $\begin{array}{l}\text { Nathalie } \\
\text { B. }\end{array}$ & BM & $\begin{array}{l}\text { Apr } 1512: 17 \\
1994\end{array}$ & purchasing & $\mathrm{nn}$ & pu-ch--ing & $2 / 5$ & $20 / 35$ \\
\hline 7 & $\begin{array}{l}\text { Nathalie } \\
\text { B. }\end{array}$ & BM & $\begin{array}{l}\text { Apr } 1512: 17 \\
1994\end{array}$ & tastes & n- & ----es(ni) & $1 / 4$ & $20 / 35$ \\
\hline 8 & $\begin{array}{l}\text { Nathalie } \\
\text { B. }\end{array}$ & BM & $\begin{array}{l}\text { Apr } 1512: 18 \\
1994\end{array}$ & niches & $\mathrm{nn}$ & ni- -- s & $1 / 4$ & $20 / 35$ \\
\hline 9 & $\begin{array}{l}\text { Nathalie } \\
\text { B. }\end{array}$ & BM & $\begin{array}{l}\text { Apr } 15 \text { 12:18 } \\
1994\end{array}$ & purchasing & + & purchasing & $3 / 5$ & $25 / 35$ \\
\hline
\end{tabular}

Les limites quantitatives d'un tel article ne permettent pas de dépasser le stade des quelques réflexions qui précèdent. Ces remarques fugitives ne visent qu'à suggérer tout le parti que l'on peut tirer de telles données et à souligner que l'on peut utiliser relativement aisément ces données «texte » en les important dans une feuille d'analyse, dans un logiciel de type tableur ou encore dans une base de données. Un filtrage croisant différents champs de données sera riche de renseignements à tous égards. Le reste est affaire de patience et d'organisation d'esprit...

\section{Conclusion}

Aujourd'hui : un développement d'une ampleur renouvelée se dessine dans notre paysage de travail, l'arrivée en masse des CD-Roms, du multimédia, de l'hypertextualité sur le marché en général et celui du logiciel de langues en particulier, demande plus que jamais que l'on fasse avancer nos travaux respectifs en comparant nos expériences. Un colloque spécialement consacré à ces problèmes devrait être rapidement organisé.

Notre réflexion doit avoir deux buts : préciser notre propre approche de l'environnement pédagogique actuel et «futur immédiat» que nous recherchons, mais aussi faire connaître nos conclusions et nos desiderata aux professionnels du logiciel. Ils ont des moyens financiers, matériels, et en connaissances informatiques qui dépassent largement toutes nos possibilités, aussi pertinentes et inventives qu'elles puissent être, mais ils sont 
dans le même temps demandeurs de critères de choix valables pour leur production, ne serait-ce que pour mieux vendre. Il faut bien se dire que, de toute façon, avec ou sans nos suggestions, ils « feront du logiciel », alors que ce soit en accord avec notre recherche.

Le risque est grand aujourd'hui de penser que toutes ces nouvelles possibilités de l'informatique font que la maîtrise de cet outil nous a désormais échappé. Le travail décrit ici montre qu'il n'en est rien. Au contraire, même, il est plus facile que jamais de monter un ensemble pédagogique intéressant avec du son, de l'image et un peu de savoir et de savoir-faire. Les utilisateurs d'Hypercard sur Macintosh savent bien depuis longtemps tout ce qui est faisable par quelqu'un d'un peu inventif avec un tel outil, il est bon de savoir qu'aujourd'hui, il est possible de faire tout aussi bien avec un PC, sous Windows.

Si le champ de réflexion est large, il est aussi accessible et attirant plus qu'il ne l'a jamais été. Il nous semble que l'EAO avec toutes les critiques qu'on pouvait lui opposer: de limitation de liberté de l'apprenant, de l'enseignant, de la matière travaillée... est aujourd'hui mort. Ou plutôt : il est en train de renaître tellement différent de ce qu'il a été qu'on ne le reconnaît déjà plus. Alors, ne perdons pas le contact, et faisons en sorte que les véritables intérêts de notre travail soient mieux compris et pris en compte.

\section{ANNEXES}

\section{Annexe 1 : Publicité}

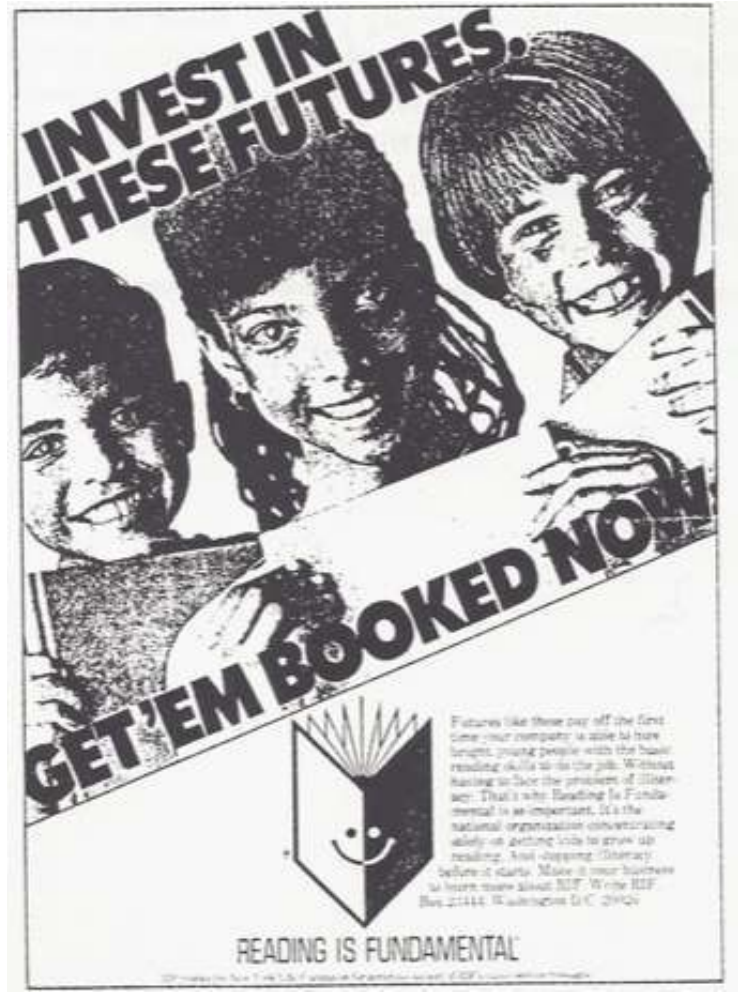

Annexe 2 : Exemple de notation 
Exemple de notation accessible à tout instant par l'apprenant. Le taux de réussite de $84 \%$ vient du fait que les réponses de type « pendu » ont été entrées avec des succès divers.

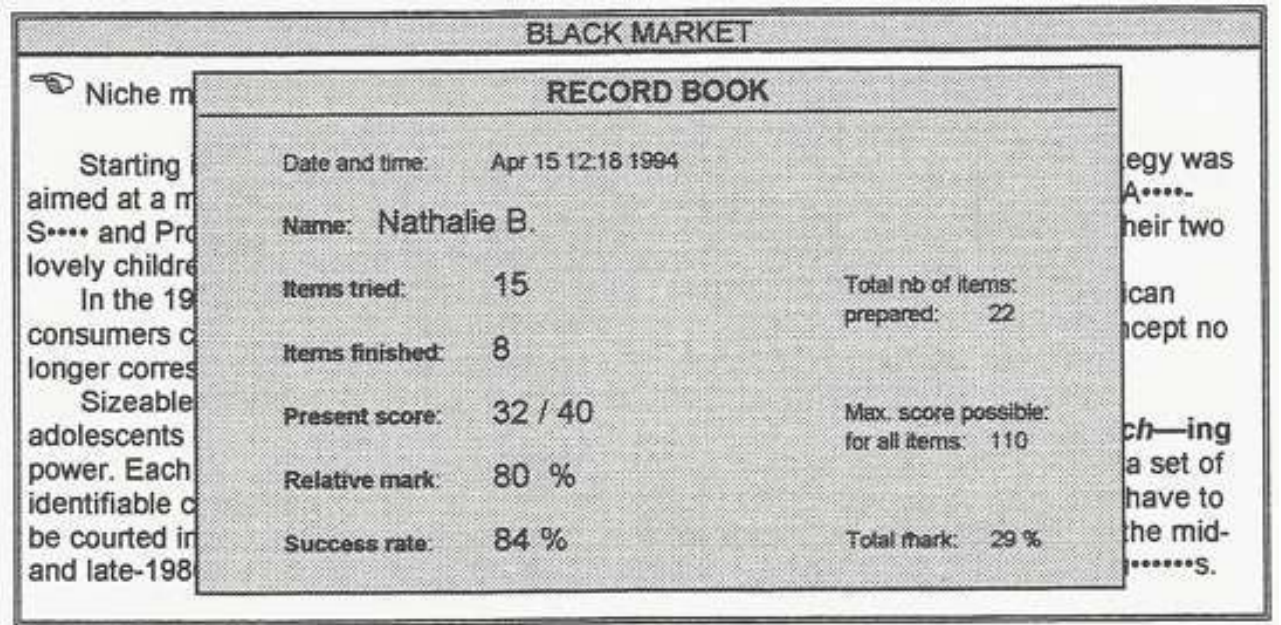

\section{NOTES}

1. Nous n'entendons pas ici le terme « acquisition » dans l'acception proposée par S. Krashen, qui insiste davantage sur le caractère « naturel » de cette notion.

2. Michèle Rivas fut longtemps directrice du Cerlaca-Dauphine et continue à inspirer de très bonnes idées à ceux qui continuent à avoir la chance et l'avantage de la côtoyer.

3. Alain Cazade a été pendant de longues années formateur académique en informatique pédagogique dans l'Académie de Créteil et s'est retrouvé responsable pédagogique du centre académique de formation « lourde » avant d'être recruté par Dauphine sur un poste de maître de conférences d'anglais.

4. Voir précision au point 6 infra, quant aux possibilités de réponse à de telles questions sur des parties de mots.

5. L'apprenant commence alors sa réponse par un trait d'union :-, ce qui représente à nos yeux un marquage spécifique suffisant pour ne pas encourir le risque cité en début de ce point 6 .

6. Voir l'extrait d'un fichier de ce type, inclus infra, juste avant la conclusion de cet article.

7. L'écran ci-dessus représente aussi fidèlement que possible l'écran original. Les représentations suivantes seront simplifiées pour faciliter la lisibilité.

8. Voir la publicité intitulée « INVEST IN THESE FUTURES - GET 'EM BOOKED NOW » en annexe 1.

9. Il faut préciser que les italiques marquant les éléments acceptés sont moins visibles ici que sur l'écran d'origine.

10. Il est possible en effet de traiter les questions dans le désordre le plus complet, et même de commencer à traiter un item à un moment donné puis d'y revenir bien plus tard, après avoir, entre temps, traité de nombreuses autres questions ou/et effectué différentes recherches (cf. lignes 5 à 9 du tableau). 


\section{RÉSUMÉS}

L'environnement hypertexte et le multimédia accompagnent aujourd'hui tout apprentissage des langues sur ordinateur. Mais cette richesse ne doit exclure ni facilité ni simplicité d'emploi tant pour l'enseignant que pour l'apprenant. L'analyse des réponses de l'apprenant et de ses tâtonnements orientera une recherche très intéressante sur les différents comportements cognitifs rencontrés. «Black Market» est un exemple d'hypertexte faisant travailler sur des textes, des documents iconographiques et des «fichiers son ». Le monde de l'EAO, en pleine reconversion, nous oblige à réfléchir ensemble à ce qu'il faut en faire pour aboutir à autre chose qu'un gadget sophistiqué ne tenant pas suffisamment compte des préoccupations légitimes de la pédagogie en général et des avancées particulières de la recherche en matière d'étude des langues.

Hypertext and multimedia equipment are essential components of today's computer-assisted language learning experience. This environment should, above all, be synonymous with simplicity and ease of use for both the teacher and learner. Facilities for retrieving and analysing students' production, including partial and/or incorrect answers, will enable CALL researchers to pursue work on learners' cognitive strategies. "Black Market" is an example of hypertext which uses sound and graphics, together with text. The world of CALL is changing rapidly. Technological advances necessitate greater cooperation among practitioners in order to avoid the danger of producing teaching materials that are nothing more than sophisticated gadgets, illsuited to the needs of teaching and research.

INDEX

Keywords : CALL, cognitive approach, hypertext

Mots-clés : approche cognitive, EAO, hypertexte, multimédia

\section{AUTEURS}

\section{ALAIN CAZADE}

Alain Cazade enseigne à l'Université Paris-IX Dauphine. alain.cazade@dauphine.fr

\section{EVELYN PERRY}

Evelyn Perry enseigne à l'IUFM Paris. 